TRANSACTIONS OF THE

AMERICAN MATHEMATICAL SOCIETY

Volume 355, Number 2, Pages 551-566

$\mathrm{S} 0002-9947(02) 03180-\mathrm{X}$

Article electronically published on October 9, 2002

\title{
BASE LOCI OF LINEAR SERIES ARE NUMERICALLY DETERMINED
}

\author{
MICHAEL NAKAMAYE
}

\begin{abstract}
We introduce a numerical invariant, called a moving Seshadri constant, which measures the local positivity of a big line bundle at a point. We then show how moving Seshadri constants determine the stable base locus of a big line bundle.
\end{abstract}

\section{INTRODUCTION}

Suppose $X$ is a smooth projective variety defined over an algebraically closed field of characteristic zero, and suppose $L$ is a line bundle on $X$. If $x \in X$, the Seshadri constant $\epsilon(x, L)$ measures the numerical positivity of $L$ at $x$. When $L$ is nef, $\epsilon(x, L)$ carries local geometric information at $x$; namely, it measures which order jets $L$ generates at $x$ asymptotically. When $L$ is not nef, however, $\epsilon(x, L)$ often ceases to carry geometrically meaningful information. In order to remedy this situation we study a slight variant of the Seshadri constant, $\epsilon_{m}(x, L)$, which we call the moving Seshadri constant of $L$ at $x$ and which specifically measures which order jets a high multiple of $L$ generates at $x$. The main result of this paper is to generalize the results of [N], establishing that the base locus of a small perturbation of a big line bundle $L$ is completely determined by the constants $\epsilon_{m}(x, L)$.

We begin by recalling the basic definitions and results of $[\mathrm{N}]$.

Definition 0.1. Suppose $L$ is a line bundle on a smooth projective variety $X$. Let

$\operatorname{BS}(L)=\left\{x \in X: s(x)=0\right.$ for all sections $s \in H^{0}(X, n L)$ and for all $\left.n>0\right\}$.

We call $\mathrm{BS}(L)$ the stable base locus of $L$.

Note that $\mathrm{BS}(L)$ is empty if and only if some positive multiple of $L$ is generated by global sections. If $D \in \operatorname{Div}(X) \otimes \mathbf{Q}$ is a divisor with rational coefficients, then Definition 0.1 can be slightly reformulated:

$$
\mathrm{BS}(L(-D))=\mathrm{BS}(k L(-k D)),
$$

where $k D \in \operatorname{Div}(X)$. This definition is independent of $k$.

Definition 0.2. Suppose $L$ is a nef line bundle on $X$. Let

$$
L^{\perp}=\left\{P \in X: c_{1}(L)^{\operatorname{dim} V} \cap V=0 \text { for some } V \subset X \text { containing } P\right\} .
$$

Received by the editors January 16, 2002.

2000 Mathematics Subject Classification. Primary 14J17.

Partially supported by NSF Grant DMS 0070190. 
We established in $[\mathrm{N}]$ that $L^{\perp}$ is Zariski closed. The main theorem of $[\mathbb{N}]$ is the following:

Theorem 0.3. Suppose $X$ is a smooth projective variety of dimension $d \geq 2$ and $L$ a big and nef line bundle on $X$. Suppose $A$ is an ample line bundle on $X$. Then there exists $\epsilon>0$ such that

$$
L^{\perp}=\operatorname{BS}(L(-\delta A))
$$

whenever $0<\delta<\epsilon$.

Thus the base locus of a nearly big and nef line bundle is determined numerically. Here we push this analysis further and show that at each stage where the base locus jumps, there is a numerical reason for the jump which can be captured by studying moving Seshadri constants, which we define here:

Definition 0.4. Suppose $X$ is a smooth projective variety and $L$ a big line bundle on $X$. For all $n \gg 0$, let $\pi_{n}: X_{n} \rightarrow X$ be a resolution of $\mathcal{I}_{B_{n}}$ where $B_{n}$ is the base locus scheme of the complete linear series $|n L|$. We assume here that $X_{n}$ is smooth. Write

$$
\pi_{n}^{*}(n L) \sim M_{n}+E_{n},
$$

where $\mathcal{O}_{X_{n}}\left(-E_{n}\right)=\pi_{n}^{-1}\left(\mathcal{I}_{B_{n}}\right) \cdot \mathcal{O}_{X_{n}}$. For any point $x$ not in $\operatorname{BS}(L)$, we define the moving Seshadri constant of $L$ at $x$ by

$$
\epsilon_{m}(x, L)=\lim _{n \rightarrow \infty} \frac{\epsilon\left(x, M_{n}\right)}{n} .
$$

The invariant $\epsilon_{m}(x, L)$ measures how many leading terms can be specified at $x$, asymptotically, in the Taylor series of a section $s \in H^{0}(X, n L)$. Note that the limit in Definition 0.4 makes sense. Indeed, since

$$
\epsilon\left(x, M_{n}\right) \leq \frac{1}{\operatorname{dim}} \sqrt{c_{1}\left(M_{n}\right)^{\operatorname{dim} X}}, \quad \forall n,
$$

and

$$
\frac{c_{1}\left(M_{n}\right)^{\operatorname{dim} X}}{n^{\operatorname{dim} X} \operatorname{dim} X !} \leq \lim _{m \rightarrow \infty} \frac{h^{0}(X, m n L)}{(m n)^{\operatorname{dim} X}}
$$

it follows that $a=\limsup _{n \rightarrow \infty}\left\{\frac{\epsilon\left(x, M_{n}\right)}{n}\right\}$ exists. Suppose there were an infinite sequence $\left\{n_{1}, n_{2}, \ldots\right\}$ such that

$$
\lim _{m \rightarrow \infty} \frac{\epsilon\left(x, M_{n_{m}}\right)}{n_{m}}=b<a .
$$

By (0.5), we can choose a positive integer $k$ such that

$$
\epsilon\left(x, M_{k}\right)>b \text {. }
$$

For any $r$ we can write $n_{r}=c k+d, d<k$. Using the fact that

$$
\frac{\epsilon\left(x, M_{c k+d}\right)}{c k+d} \geq \frac{\epsilon\left(x, c M_{k}\right)+\epsilon\left(x, M_{d}\right)}{c k+d},
$$

we find that

$$
\epsilon\left(x, M_{n_{r}}\right) \geq \frac{c \epsilon\left(x, M_{k}\right)+\epsilon\left(x, M_{d}\right)}{c k+d} .
$$

Since $d$ is bounded and $c \rightarrow \infty$ as $r \rightarrow \infty$, this contradicts (0.6).

Next, we isolate those line bundles where the stable base locus changes. 
Definition 0.7. Suppose $D$ is a $\mathbf{Q}$-divisor on $X$ in the interior of the effective cone, and that $A$ is an ample $\mathbf{Q}$-divisor. Suppose $\alpha$ is a positive real number. We call $\alpha$ a jumping number relative to $D$ and $A$ if $\operatorname{BS}\left(D-\alpha A+\epsilon_{1} A\right)$ is properly contained in $\operatorname{BS}\left(D-\alpha A-\epsilon_{2} A\right)$ for any $\epsilon_{1}, \epsilon_{2}>0$ such that $\alpha+\epsilon_{1}$ and $\alpha-\epsilon_{2}$ are rational numbers.

Intuitively, the stable base locus of $D-\beta A$ changes each time $\beta$ passes through a jumping value $\alpha$. Our main theorem shows that jumping numbers are determined by the relevant moving Seshadri constants approaching zero:

Theorem 0.8. Suppose $E$ is a big $\mathbf{Q}$-divisor on a smooth projective variety $X$, and $A$ is an ample divisor. Suppose $\alpha$ is a jumping number relative to $E$ and $A$. We will write

$$
D=E-\alpha A .
$$

Let $Y$ be an irreducible component of $\operatorname{BS}(D-\beta A)$ for $0<\beta<\epsilon$ such that $Y$ is not in $\operatorname{BS}(D+\beta A)$ for any $\beta>0$. Then for a general point $\eta \in Y$,

$$
\lim _{\beta \rightarrow 0} \epsilon_{m}(\eta, D+\beta A)=0
$$

here the limit is of course from above, since $\epsilon_{m}(\eta, D+\beta A)$ has not been defined for $\beta \leq 0$.

Note the similarity between Thereom 0.8 and Theorem 0.3 . In both cases, the critical stage where the base locus changes is marked by the appropriate Seshadri constants approaching zero. The proof of Theorem 0.8 is more subtle than that of Theorem 0.3 because the divisors $D+\beta A$, as $\beta$ approaches 0 , are not nef and their base loci cannot necessarily all be resolved on a single birational model of $X$. Moreover, and more seriously, the critical number $\alpha$ may well be irrational, making it impossible to actually consider a limit linear series. The proof of Theorem 0.8 nonetheless follows along the same lines as the proof of Theorem 0.3 , namely by lifting a non-vanishing section on a subscheme supported on $Y$ using a cohomological vanishing theorem. In order to avoid working on infinitely many birational models of $X$, we examine and compare carefully the base locus scheme of $n(D+\beta A)$ for $\beta$ slightly less than 0 and for $\beta$ slightly greater than 0 . Theorem 0.8 amounts to a type of "continuity" of these base loci.

The outline of this paper is as follows. In $\S 1$ we prove Theorem 0.8 The ideas employed in the proof are very close to the work of Demailly, Ein, and Lazarsfeld DEL on asymptotic multiplier ideals, but the key technical lemmas require other limiting procedures, and we were unable to obtain these results mechanically from DEL]. The fundamental idea is simply to keep track of how the base loci of $|n D|$ vary under small perturbations and how cohomology groups on these base loci vary. The key insight is to restrict ourselves to a very specific vector space of sections, namely those generating specific jets at a general point $\eta \in Y$. In $\S 2$ we will apply the techniques developed in $\S 1$ to prove a theorem of Fujita, also obtained in DEL, in a very concrete fashion which should be helpful in many applications.

Our original goal in this work was to obtain a sufficiently deep understanding of stable base loci and the effective cone in order to derive certain consequences in diophantine geometry. The present work comes close, but needs to be made more effective before yielding the desired corollaries. Nevertheless, the problem of understanding the effective cone, and defining numerical invariants on it, seems 
sufficiently interesting in its own right to be pursued. In particular, if one defines $\epsilon_{m}(x, L)$ to be zero whenever $x \in \mathrm{BS}(L)$, then $\epsilon_{m}(x, L)$ can be viewed as a function on the rational classes in the cone of divisors modulo numerical equivalence: in this context, the content of Theorem 0.8 is to establish that this function is continuous with respect to $L$.

Notation and Conventions

- If $\mathcal{F}$ is a coherent sheaf on $X$, then $h^{i}(X, \mathcal{F})=\operatorname{dim} H^{i}(X, \mathcal{F})$.

- If $\mathcal{I} \subset \mathcal{O}_{X}$ is an ideal sheaf, then we let $Z(\mathcal{I}) \subset X$ denote the associated subscheme of zeroes of $\mathcal{I}$. If $f$ is a rational function on $X$, we denote by $Z(f)$ the divisor of zeroes of $f$.

- If $D$ is a Cartier divisor on $X$, we will often abbreviate $H^{0}\left(X, \mathcal{O}_{X}(D)\right)$ by $H^{0}(X, D)$.

- If $V \subset X$ is a subvariety, we denote by $\mathcal{I}_{V}^{a}$ the ideal sheaf of functions whose order of vanishing along $V$ is at least $a$. Similarly we will write $a V$ for the scheme defined by the ideal sheaf $\mathcal{I}_{V}^{a}$.

- If $Y$ is a scheme, we denote by $Y_{\text {red }}$ the reduced scheme with support equal to the support of $Y$.

- For a variety $X$, we let $K(X)$ denote the field of rational functions on $X$.

\section{Continuity of Seshadri Constants}

Choose an $\epsilon>0$ so that $Y$ is an irreducible component of $\operatorname{BS}(D-\beta A)$ for all $0<\beta \leq \epsilon$. We also assume that $D-\epsilon A \in \operatorname{Div}(X) \otimes \mathbf{Q}$. Next choose an $n>0$ so that $n(D-\epsilon A)$ is a genuine line bundle whose sections give a birational map to projective space. Let

$$
\pi: X^{\prime} \rightarrow X
$$

be a birational map, with $X^{\prime}$ smooth, such that

$$
\pi^{*}(n(D-\epsilon A)) \sim M_{n}+E_{n},
$$

where $M_{n}$ is generated by global sections and $\pi^{-1} \mathcal{I}_{B} \cdot \mathcal{O}_{X^{\prime}}=\mathcal{O}_{X^{\prime}}\left(-E_{n}\right)$ with $B$ denoting the base locus scheme of $|n(D-\epsilon A)|$. Since $M_{n}$ is big and nef, we have

$$
h^{1}\left(X^{\prime}, k M_{n}\right) \leq O\left(k^{\operatorname{dim} X-1}\right) .
$$

Let $Z_{n}$ be the zero scheme of $\pi_{*} \mathcal{O}_{X^{\prime}}\left(-E_{n}\right)$ and let $k Z_{n}$ denote the zero scheme of the ideal sheaf

$$
\pi_{*} \mathcal{O}_{X^{\prime}}\left(-k E_{n}\right) \subset \mathcal{O}_{X} .
$$

Note that by [EV], 3.3, $Z_{n}$ does not depend on the choice of resolution $\pi$. Using the long exact cohomology sequence associated to the exact sequence of sheaves

$$
\begin{aligned}
0 \rightarrow \mathcal{I}_{k Z_{n}} \otimes \mathcal{O}_{X}(k n(D-\epsilon A)) & \rightarrow \mathcal{O}_{X}(k n(D-\epsilon A)) \\
& \rightarrow \mathcal{O}_{k Z_{n}}(k n(D-\epsilon A)) \rightarrow 0
\end{aligned}
$$

gives

$$
\begin{aligned}
H^{0}\left(X, \mathcal{O}_{X}(k n(D-\epsilon A))\right) & \rightarrow H^{0}\left(k Z_{n}, \mathcal{O}_{X}(k n(D-\epsilon A))\right) \\
& \rightarrow H^{1}\left(X, \mathcal{O}_{X}\left(k n(D-\epsilon A) \otimes \mathcal{I}_{k Z_{n}}\right)\right) .
\end{aligned}
$$

By (1.1), pushed down to $X$,

$$
h^{1}\left(X, \mathcal{O}_{X}\left(k n(D-\epsilon A) \otimes \mathcal{I}_{k Z_{n}}\right)\right)=O\left(k^{\operatorname{dim} X-1}\right),
$$


and thus most sections of $H^{0}\left(k Z_{n}, \mathcal{O}_{X}(k n(D-\epsilon A))\right)$ lift to $X_{n}$. In order to prove Theorem 0.8 we will show that if

$$
\lim _{\beta \rightarrow 0} \epsilon_{m}(\eta, D+\beta A) \neq 0,
$$

then it is possible to use (1.2) to lift to $X$ a section of $H^{0}\left(k Z_{n}, \mathcal{O}_{X}(k n(D-\epsilon A))\right)$ with small order of vanishing along $Y$. On the other hand, we will establish that all sections of $H^{0}\left(X, \mathcal{O}_{X}(k n(D-\epsilon A))\right)$ have large order of vanishing along $Y$, thus deriving a contradiction.

Since $\epsilon_{m}(\eta, D+\beta A)$ is a decreasing function of $\beta$, if Theorem 0.8 were false, there must exist a $\delta>0$ such that

$$
\epsilon_{m}(\eta, D+\beta A)>\delta, \quad \forall \beta>0
$$

We will use the following four results in the proof of Theorem 0.8

Lemma 1.4. With notation as above, there exists $\gamma>0$, not depending on $\epsilon$ or $n$, such that

$$
\mathcal{I}_{Z_{n}} \subset \mathcal{I}_{Y}^{\gamma \epsilon n}, \quad \forall n \text { sufficiently divisible, }
$$

and, in fact, $\mathcal{I}_{k Z_{n}} \subset \mathcal{I}_{Y}^{\gamma \epsilon k n}$ for all $k>0$.

Lemma 1.4 simply states that once a subvariety $Y$ enters the stable base locus of $D$, multiplicity along $Y$ must grow linearly as $D$ moves away from the ample cone. This result is a formal consequence of properties of multiplier ideal sheaves when phrased in the language of [DEL]; we give an alternative proof using [ELN].

Write

$$
Z_{n}=Y_{n} \cup W_{n},
$$

where $Y_{n}$ is supported on $Y$ and $\operatorname{supp}\left(W_{n}\right)$ does not contain $Y$. To avoid ambiguity, one can choose $\mathcal{I}_{W_{n}}$ maximal over all such possible expressions; in other words, the local primary decomposition of $\mathcal{I}_{W_{n}}$ should have no embedded components supported on $\operatorname{supp}(Y)$. In order to prove Theorem 0.8 , we will need to consider the ideal sheaves $\mathcal{I}_{Z_{n}}$ as depending on $\epsilon$ and then take the limit as $\epsilon \rightarrow 0$. We will write $Z_{n}(\epsilon), Y_{n}(\epsilon)$, and $W_{n}(\epsilon)$ in this case, including the possibility that $\epsilon<0$; note that $n$ actually depends upon $\epsilon$, since it is the multiple used to clear denominators in $\mathbf{Q}$ divisors.

Let $\phi: V \rightarrow X$ be a resolution of the base locus of $|n(D+\epsilon A)|$ with exceptional divisor $E$, so that $\mathcal{I}_{Z_{n}(-\epsilon)}=\phi_{*} \mathcal{O}_{V}(-E)$ by definition.

Lemma 1.6. Assume that $\gamma \epsilon<\delta$. For all $k \gg 0$ and for all $n$ sufficiently large, there is a vector subspace

$$
W_{k, n}(\epsilon) \subset H^{0}\left(k Y_{n}(\epsilon), k n(D+\epsilon A) \otimes \mathcal{I}_{k Z_{n}(-\epsilon)}\right)
$$

with

$$
\operatorname{dim}\left(W_{k, n}(\epsilon)\right) \geq O\left((\gamma \epsilon n k)^{\operatorname{codim}(Y, X)}(\delta n k)^{\operatorname{dim} Y}\right)
$$

and such that no nonzero section $s \in W_{k, n}$ vanishes to order $\geq \gamma \epsilon k n$ along $Y$. Moreover, the implied constant depends only on $X$.

Lemma 1.6 merely states that there will exist a large space of functions on $Y$ which vanish along $k Z_{n}(-\epsilon)$; this is clear, since we have a lower bound on the Seshadri constant of $D+\epsilon A$ along a generic point of $Y$, independent of $\epsilon$, and so this space of sections will come from restricting sections of $H^{0}(X, k n(D+\epsilon A))$ 
which generate appropriate jets at $\eta$. For the following Corollary, we assume for simplicity that $D \in \operatorname{Div}(X) \otimes \mathbf{Q}$ and hence that $\epsilon \in \mathbf{Q}$. We will show how to avoid this assumption in the proof of Theorem 0.8 .

Corollary 1.7. With the same notation as in Lemma 1.6, let $B$ be an ample line bundle on $X$ and for $a \in(0,1) \cap \mathbf{Q}$, let $V_{k, n}(a \epsilon) \subset W_{k, n}(a \epsilon)$ be defined by

$$
V_{k, n}(a \epsilon)=\left\{s \in W_{k, n}(a \epsilon) \mid Z(s)-a k n B \text { is effective }\right\} .
$$

Then there exists an integer $n$ such that for all $k \gg 0$,

$$
\operatorname{dim}\left(V_{k, n}(a \epsilon)\right) \geq O\left((\gamma a \epsilon n k)^{\operatorname{codim}(Y, X)}(\delta n k)^{\operatorname{dim} Y}\right)-O\left(a^{\operatorname{codim}(Y, X)+1}(n k)^{\operatorname{dim} X}\right),
$$

with implied constants depending only on $X, \epsilon$, and $B$.

Corollary 1.7 examines what happens to the subspace of sections of Lemma 1.6 when we replace the divisor $k n(D+\epsilon A)$ with the less positive divisor $k n(D-\epsilon A)$, the idea being to limit how many of the sections of Lemma 1.6 can be lost.

For our final Lemma, we adopt the following notational conventions. We let $n$ denote an integer to be fixed in the proof. For each positive integer $m$ we let

$$
\begin{aligned}
& n_{m}=2^{m} r_{m} n, \\
& n_{m}^{\prime}=\left(2^{m}-1\right) r_{m} n,
\end{aligned}
$$

where $r_{m}$ is a positive integer to be specified in the proof.

Lemma 1.8. Fix a number $0<\beta<\epsilon$ so that $D-\beta A \in \operatorname{Div}(X) \otimes \mathbf{Q}$. There exists an ideal sheaf $\mathcal{J}$ on $X$, with $Y$ not contained in $Z(\mathcal{J})$, such that for all $k$ sufficiently large and all $m \geq 0$,

$$
\mathcal{J}^{\frac{k n_{m}}{2^{m}}} \cdot \mathcal{I}_{k Z_{n_{m}^{\prime}}(-\beta)} \subset \mathcal{I}_{k W_{n_{m}}\left(-\beta+\frac{\epsilon}{2^{m}}\right)} .
$$

Moreover, if $D \in \operatorname{Div}(X) \otimes \mathbf{Q}$, then this relation also holds for $\beta=0$.

Having moved from $k n(D+\epsilon A)$ to $k n(D-\epsilon A)$ with Corollary 1.7 we now use Lemma 1.8 to replace $\mathcal{I}_{k Z_{n}(-\epsilon)}$ with $\mathcal{I}_{k W_{n}(\epsilon)}$ in Lemma 1.6. Combined with the previous results and the exact sequence (1.2), this will exhibit a large vector space of sections of $H^{0}(X, k n(D-\epsilon A))$ having small order of vanishing along $Y$, contradicting Lemma 1.4 More specifically,

Proof of Theorem 0.8 . We will assume, for simplicity, that $D \in \operatorname{Div}(X) \otimes \mathbf{Q}$, and show how to remove this hypothesis at the end. Applying Lemma 1.8 for $\beta=\frac{\epsilon}{2^{m}}$, and replacing $\mathcal{J}$ by $\mathcal{J}^{2}$, gives an ideal sheaf $\mathcal{J}$ satisfying, for $k$ sufficiently divisible relative to $m$,

$$
\mathcal{J}^{\frac{k n_{m}}{2^{m}}} \cdot \mathcal{I}_{k Z_{n_{m}^{\prime}}\left(-\frac{\epsilon}{2^{m}}\right)} \subset \mathcal{I}_{k W_{n_{m}}\left(\frac{\epsilon}{2^{m}}\right)}, \quad \forall m \geq 0 .
$$

Choose a line bundle $C$ on $X$ so that $C \otimes \mathcal{J}$ is generated by global sections. We let

$$
B=C+2 \epsilon A \text {. }
$$

Applying Corollary 1.7 with $a=\frac{1}{2^{m}}$ gives a subspace

$$
U_{k, n_{m}^{\prime}}(a \epsilon) \subset H^{0}\left(k Y_{n_{m}^{\prime}}(a \epsilon), k n_{m}^{\prime}(D+a \epsilon A) \otimes \mathcal{I}_{k Z_{n_{m}^{\prime}}}(-a \epsilon)\right)
$$


with bounded dimension:

$$
\begin{aligned}
\operatorname{dim}\left(U_{k, n_{m}^{\prime}}\right)(a \epsilon) \geq & O\left(\left(\gamma a \epsilon n_{m}^{\prime} k\right)^{\operatorname{codim}(Y, X)}\left(\delta n_{m}^{\prime} k\right)^{\operatorname{dim} Y}\right) \\
& -O\left(a^{\operatorname{codim}(Y, X)+1}(n k)^{\operatorname{dim} X}\right) .
\end{aligned}
$$

Since all sections in $U_{k, n_{m}^{\prime}}(a \epsilon)$ are defined via restriction from sections on $X$ and since none of these sections vanishes on $\gamma a \epsilon k n_{m}^{\prime} Y$, we can consider

$$
U_{k, n_{m}^{\prime}}(a \epsilon) \subset H^{0}\left(k Y_{n_{m}}(a \epsilon), k n_{m}^{\prime}(D+a \epsilon A) \otimes \mathcal{I}_{k Z_{n_{m}^{\prime}}}(-a \epsilon)\right) .
$$

By hypothesis, every section $s \in U_{k, n_{m}^{\prime}}(a \epsilon)$ vanishes along $B=C+2 \epsilon A$ to multiplicity at least $a k n_{m}^{\prime}$; removing the base divisor $B$ gives a vector space

$$
V_{k, n_{m}^{\prime}}(a \epsilon) \subset H^{0}\left(k Y_{n_{m}}(a \epsilon), k n_{m}^{\prime}(D-a \epsilon A-a C) \otimes \mathcal{I}_{k Z_{n_{m}^{\prime}}}(-a \epsilon)\right) .
$$

Note that removing the base divisor $B$ does not effect membership in the ideal sheaf $\mathcal{I}_{k Z_{n_{m}^{\prime}}}(-a \epsilon)$. Tensoring the vector space $V_{k, n_{m}^{\prime}}(a \epsilon)$ by $a k n_{m}^{\prime} C$ and using (1.9.1), taking care again to replace $\mathcal{J}$ with $\mathcal{J}^{2}$ to account for the difference between $n_{m}$ and $n_{m}^{\prime}$, allows us to view $V_{k, n_{m}^{\prime}}(a \epsilon)$ as

$$
V_{k, n_{m}^{\prime}}(a \epsilon) \subset H^{0}\left(k Y_{n_{m}}(a \epsilon), k n_{m}^{\prime}(D-a \epsilon A) \otimes \mathcal{I}_{k W_{n_{m}}}(a \epsilon)\right) .
$$

Note that, since $\eta \notin \operatorname{supp}(C)$, no section of $V_{k, n_{m}^{\prime}}(a \epsilon)$ has multiplicity greater than $\gamma \epsilon a k n_{m}^{\prime}$ at $\eta$. We now replace $\gamma$ with $\gamma / 2$ and choose a section

$$
\left.\sigma \in H^{0}\left(X, k\left(n_{m}-n_{m}^{\prime}\right)(D-a \epsilon A)\right)\right)
$$

of minimal index at $\eta$, and tensor to obtain an inclusion

$$
V_{k, n_{m}^{\prime}}(a \epsilon) \stackrel{\otimes \sigma}{\hookrightarrow} H^{0}\left(k Y_{n_{m}}(a \epsilon), k n_{m}(D-a \epsilon A) \otimes \mathcal{I}_{k W_{n_{m}}}(a \epsilon)\right) .
$$

For $m$ sufficiently large, every section in the vector subspace (1.9.3) has multiplicity at most $\gamma a \epsilon k n_{m}$ at $\eta$ : here we use the extra $\gamma / 2$ obtained by replacing $\gamma$ by $\gamma / 2$.

Using (1.9.2), we see that for $m$ sufficiently large,

$$
\operatorname{dim}\left(V_{k, n_{m}^{\prime}}\right) \geq O\left((\gamma a \epsilon n k)^{\operatorname{codim}(Y, X)}(\delta n k)^{\operatorname{dim} Y}\right)
$$

But by (1.5) any nonzero section $s$ in the vector subspace (1.9.3) glues together with the zero section on $k W_{n_{m}}(a \epsilon)$ to give a nonzero section of

$$
H^{0}\left(k Z_{n_{m}}(a \epsilon), k n_{m}(D-a \epsilon A)\right) .
$$

Using (1.2), however, we obtain, for $m$ sufficiently large, many nonzero sections of

$$
H^{0}\left(X, k n_{m}(D-a \epsilon A)\right)
$$

with order of vanishing strictly less than $\gamma a \epsilon k n_{m}$ along $Y$. This contradicts Lemma 1.4, or more precisely (1.4.3), and establishes Theorem 0.8 when $D \in \operatorname{Div}(X) \otimes \mathbf{Q}$.

Suppose now that $D$ is only a real divisor and consequently that $\epsilon$ is irrational; in particular, Corollary 1.7 does not hold as stated and Lemma 1.8 does not hold for $\beta=0$. The same proof holds, except that we need to employ an additional limiting procedure. First, for (1.9.1), which no longer makes any sense, we choose

$$
\beta_{m}, \gamma_{m} \in\left(\frac{\epsilon}{2^{m+1}}, \frac{\epsilon}{2^{m}}\right)
$$


so that $D-\beta_{m} A, D+\gamma_{m} A \in \operatorname{Div}(X) \otimes \mathbf{Q}$. Then for $k$ sufficiently divisible with respect to $m$ we have

$$
\mathcal{J}^{\frac{k n}{2^{m}}} \cdot \mathcal{I}_{k Z_{n_{m}^{\prime}}\left(-\beta_{m}\right)} \subset \mathcal{I}_{k W_{n_{m}}\left(\gamma_{m}\right)}, \quad \forall m \geq 0 .
$$

As far as Corollary 1.7 goes, the problem is that $W_{k, n_{m}}(a \epsilon)$ will not make sense in general, because $D+a \epsilon A$ may not be a rational class. This is not a problem, however, since for any rational class $D+b A$ with $b>a \epsilon$, the estimate of Corollary 1.7 will still hold; so the same limiting procedure applies.

Proof of Lemma 1.4 Suppose $\phi: V \rightarrow X$ is a birational map with $V$ smooth, and suppose that there is a unique smooth exceptional divisor $E_{Y}$ dominating $Y$ : this can be obtained, for example, by taking an embedded resolution of $Y$ in $X$ followed by a blow-up of the strict transform of $Y$. Consider the commutative diagram

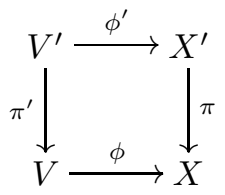

Setting $E_{n}^{\prime}=\left(\phi^{\prime}\right)^{*} E_{n}$, we claim that it is sufficient to show that

$$
E_{n}^{\prime}-\left(\pi^{\prime}\right)^{*} \gamma \epsilon n \tilde{E}_{Y} \text { is effective. }
$$

To see this, suppose that $\eta$ is a general point of $Y$. If, for some $k>0, \mathcal{I}_{k Z_{n}}$ were not contained in $\mathcal{I}_{Y}^{\gamma \epsilon k n}$, then there would exist an open subset $U \subset X$ and a function $f \in \Gamma\left(U, \mathcal{O}_{U}\right)$ such that $\operatorname{ord}_{\eta}(f)<\gamma \epsilon k n$ but $\left(\phi^{\prime}\right)^{*}\left(\pi^{*}(Z(f))\right)-k E_{n}^{\prime}$ is effective. This contradicts 1.4.1), however, since $\left(\phi^{\prime}\right)^{*}\left(\pi^{*} Z(f)\right)=\left(\pi^{\prime}\right)^{*}\left(\phi^{*} Z(f)\right)$ and by hypothesis

$$
\operatorname{mult}_{E_{Y}}\left(\phi^{*} Z(f)\right)<\gamma \epsilon n,
$$

and hence $\left(\pi^{\prime}\right)^{*}\left(\phi^{*} Z(f)\right)-\left(\pi^{\prime}\right)^{*} \gamma \epsilon n \tilde{E}_{Y}$ is not effective.

Choose a positive integer $b$ sufficiently large so that

$$
T_{X} \otimes b A \text { is generated by global sections. }
$$

Suppose $s \in H^{0}(X, n(D-\epsilon A))$. We claim that

$$
\operatorname{ord}_{\eta}(s) \geq \frac{n \epsilon}{b}
$$

If not, then there exists a local differential operator $D$ of order $t<\frac{n \epsilon}{b}$ such that $D(s)$ does not vanish at a general point of $Y$. By the theory of [ELN], there exists a differential section

$$
s^{\prime} \in H^{0}(X, n D-n \epsilon A+t b A)
$$

with $s^{\prime}(\eta) \neq 0$. This contradicts the fact that $Y$ is in the base locus of

$$
H^{0}(X, n D-n \epsilon A+t b A) \text {, }
$$

since $-n \epsilon+t b<0$. Thus we may take $\gamma=\frac{1}{b}$, and this clearly does not depend upon $\epsilon$ or $n$, establishing Lemma 1.4 
Proof of Lemma 1.6. Suppose $\eta \in Y$ is a general, hence smooth, point, and let $\left\{x_{1}, \ldots, x_{d}\right\}$ be a system of local parameters at $\eta$ such that $\mathcal{I}_{Y} \subset \mathcal{O}_{X}$ is generated on an open set $U \supset \eta$ by $x_{1}, \ldots, x_{c}$. Let $m_{\eta} \subset \mathcal{O}_{\eta, X}$ denote the maximal ideal of functions vanishing at $\eta$, and let

$$
U_{k, n}=\mathcal{O}_{\eta, X} / m_{\eta}^{\delta k n} .
$$

We define $V_{k, n}(\epsilon) \subset U_{k, n}$ to be the vector subspace generated by the images of the following collection of monomials in the local parameters $\left\{x_{1}, \ldots, x_{d}\right\}$ :

$$
\left\{M\left(x_{1}, \ldots, x_{c}\right) M^{\prime}\left(x_{c+1}, \ldots, x_{d}\right) \mid \operatorname{deg}(M)<\gamma \epsilon n k \text { and } \operatorname{deg}\left(M^{\prime}\right)<\delta n k\right\} .
$$

By (1.3), we know that for $n$ sufficiently large, the evaluation map followed by the projection to the quotient

$$
\pi_{k, n}: H^{0}(X, k n(D+\epsilon A)) \rightarrow U_{k, n}
$$

is surjective. Choose a vector subspace $V_{k, n}^{\prime}(\epsilon) \subset H^{0}(X, k n(D+\epsilon A))$ such that

$$
\pi_{k, n}: V_{k, n}^{\prime}(\epsilon) \rightarrow V_{k, n}(\epsilon) \text { is an isomorphism. }
$$

Note that by (1.3) we can assume that for $n$ sufficiently large, $n(D+\epsilon A)$ generates $\delta n$-jets at $\eta$, and consequently we may assume that

$$
V_{k, n}^{\prime}(\epsilon) \subset \operatorname{Sym}^{k}\left(H^{0}(X, n(D+\epsilon A))\right) .
$$

We claim that the restriction map

$$
\phi_{k, n}: V_{k, n}^{\prime}(\epsilon) \rightarrow H^{0}\left(k Y_{n}(\epsilon), k n(D+\epsilon A)\right) \text { is injective. }
$$

By Lemma 1.4 it is sufficient to show that the restriction

$$
\psi_{k, n}: V_{k, n}^{\prime}(\epsilon) \rightarrow H^{0}(\gamma \epsilon k n Y, k n(D+\epsilon A))
$$

is injective. If $\psi_{k, n}$ were not injective, there would exist a nonzero polynomial $P\left(x_{1}, \ldots, x_{d}\right)$ in the monomials (1.6.1) such that $P \in \mathcal{I}_{\gamma \epsilon k n Y}$. Choose a monomial $x^{a_{1}} \ldots x^{a_{d}}$ whose coefficient in $P$ is nonzero and such that $a_{1}+\ldots+a_{c}$ is minimal. Consider

$$
Q\left(x_{1}, \ldots, x_{d}\right)=\frac{\partial^{a_{1}}}{\partial x_{1}^{a_{1}}} \cdots \frac{\partial^{a_{c}}}{\partial x_{c}^{a_{c}}} P\left(x_{1}, \ldots, x_{d}\right) .
$$

By choice of $\left(a_{1}, \ldots, a_{c}\right)$, all terms in $Q\left(x_{1}, \ldots, x_{d}\right)$ vanish identically along $Y$ except those coming from terms of the type

$$
\frac{\partial^{a_{1}}}{\partial x_{1}^{a_{1}}} \cdots \frac{\partial^{a_{c}}}{\partial x_{c}^{a_{c}}}\left(x_{1}^{a_{1}} \ldots x_{c}^{a_{c}}\right) M^{\prime}\left(x_{c+1}, \ldots, x_{d}\right) .
$$

But no nonzero sum of functions of the type appearing in (1.6.5) can vanish identically along $Y$, since the functions $x_{c+1}, \ldots, x_{d}$ form a transcendence basis for the function field of $Y$. Hence

$$
\operatorname{ord}_{Y}\left(P\left(x_{1}, \ldots, x_{d}\right)\right) \leq a_{1}+\ldots+a_{c}<\gamma \epsilon k n,
$$

contradicting the assumption that $P \in \mathcal{I}_{\gamma \in k n Y}$.

We conclude from (1.6.2) that

$$
\operatorname{dim}\left(\phi_{k, n}\left(V_{k, n}^{\prime}(\epsilon)\right)\right) \geq \frac{(\gamma \epsilon n k)^{\operatorname{dim} Y}(\delta n k)^{\operatorname{codim}(Y, X)}}{d !} .
$$

By (1.6.3), each section $s \in V_{k, n}^{\prime}(\epsilon)$ vanishes along $Z_{n}(-\epsilon)$ to order at least $k$, and consequently vanishes along $k Z_{n}(-\epsilon)$. Thus we can take $W_{k, n}(\epsilon)=\phi_{k, n}\left(V_{k, n}^{\prime}(\epsilon)\right)$ 
in Lemma 1.6 with the implied constant being $\frac{1}{d !}$, which does not depend upon $\epsilon$, as desired; the fact that no section of $W_{k, n}(\epsilon)$ vanishes along $Y$ to order $\geq \gamma \epsilon k n$ is guaranteed by (1.6.6).

Proof of Corollary 1.7. As remarked above, in order for the statement of Corollary 1.7 to make sense, we will assume that $D \in \operatorname{Div}(X) \otimes \mathbf{Q}$ and $\epsilon \in \mathbf{Q}$. We will also write

$$
\rho=a \epsilon
$$

Assuming that $B$ is effective, and for later purposes, very ample, consider the exact sequence

$$
\begin{aligned}
& H^{0}\left(k Y_{n}(\rho), k n(D+\rho A-a B)\right) \rightarrow H^{0}\left(k Y_{n}(\rho), k n(D+\rho A)\right) \\
& \stackrel{\psi}{\longrightarrow} H^{0}\left(\operatorname{akn} B \cap k Y_{n}(\rho), k n(D+\rho A)\right) \text {. }
\end{aligned}
$$

By hypothesis, we can write

$$
\operatorname{akn} B=\bigcup_{i=1}^{a k n} B_{i}
$$

with each $B_{i}$ linearly equivalent to $B$. Let $H_{W}^{0}\left(B_{i} \cap k Y_{n}(\rho), k n(D+\rho A)\right)$ denote the image of $W_{k, n}(\rho)$, defined in Lemma 1.6, in $H^{0}\left(B_{i} \cap k Y_{n}(\rho), k n(D+\rho A)\right)$. Since $\operatorname{dim}\left(V_{k, n}(\rho)\right)=\operatorname{dim}(\operatorname{ker}(\psi))$, we have, substituting into (1.7.1),

$$
\operatorname{dim}\left(V_{k, n}(\rho)\right) \geq \operatorname{dim}\left(W_{k, n}(\rho)\right)-\sum_{i=1}^{a k n} h_{W}^{0}\left(B_{i} \cap k Y_{n}(\rho), k n(D+\rho A)\right) .
$$

By Lemma 1.6] it is sufficient, in order to establish Corollary 1.7, to prove that

$$
\sum_{i=1}^{a k n} h_{W}^{0}\left(B_{i} \cap k Y_{n}(\rho), k n(D+\rho A)\right) \leq O\left(a^{\operatorname{codim}(Y, X)+1}(n k)^{\operatorname{dim} X}\right) .
$$

Thus for a general hypersurface $B$ we need to establish that

$$
h_{W}^{0}\left(B \cap k Y_{n}(\rho), k n(D+\rho A)\right) \leq O\left(a^{\operatorname{codim}(Y, X)}(n k)^{\operatorname{dim} X-1}\right)
$$

with the implied constant depending only on $X, B$, and $\epsilon$.

We first eliminate the possibility that $\operatorname{dim}(Y)=0$. In this case, $Y=P$ is a point, disjoint from the rest of the base locus $\operatorname{BS}(D-\epsilon A)$. This is impossible, however, since (1.2) in combination with Lemma 1.4 would establish that it is possible to lift a section

$$
s^{\prime} \in H^{0}\left(k Y_{n}(\epsilon), k n(D-\epsilon A)\right)
$$

with order of vanishing $<\gamma \epsilon k n$ at $P$, violating (1.4.3).

Assume now that $\operatorname{dim} Y \geq 1$. Let $X_{k, n}(\rho)=B \cap k Y_{n}(\rho)$ and choose general divisors $D_{1}, \ldots, D_{c} \in|B|$, where $c=\operatorname{dim} Y-1$. We have an exact sequence

$$
\begin{aligned}
\left.H^{0}\left(X_{k, n}(\rho), k n\left(D+\rho A-D_{1}\right)\right)\right) & \stackrel{f_{k, n}}{\longrightarrow} H^{0}\left(X_{k, n}(\rho), k n(D+\rho A)\right) \\
& \rightarrow H^{0}\left(k n D_{1} \cap X_{k, n}(\rho), k n(D+\rho A)\right) .
\end{aligned}
$$

Since $B$ can be chosen suitably positive, we have

$$
\operatorname{Image}\left(f_{k, n}\right) \cap H_{W}^{0}\left(X_{k, n}(\rho), k n(D+\rho A)\right)=\emptyset .
$$


It follows, using the same argument as after (1.7.2), that

$$
h_{W}^{0}\left(X_{k, n}(\rho), k n(D+\rho A)\right) \leq k n h_{W}^{0}\left(X_{k, n}(\rho) \cap D_{1}, k n(D+\rho A)\right) .
$$

Proceeding inductively gives

$$
\begin{gathered}
h_{W}^{0}\left(X_{k, n}(\rho), k n(D+\rho A)\right) \\
\leq(k n)^{c} h_{W}^{0}\left(X_{k, n}(\rho) \cap D_{1} \cap \ldots \cap D_{c}, k n(D+\rho A)\right) .
\end{gathered}
$$

Since $D_{1}, \ldots, D_{c}$ are general, the intersection $D_{1} \cap \ldots \cap D_{c} \cap X_{k, n}(\rho)$ will contain only general points of $Y$, and thus (1.7.4), along with [Fu], Corollary 12.4 and Example 4.3.4, implies that

$$
h_{W}^{0}\left(B \cap k Y_{n}(\rho), k n(D+\rho A)\right) \leq(k n)^{c} \operatorname{deg}_{B}\left(Y_{\text {red }}\right) \ell_{Y}\left(\mathcal{O}_{k Y_{n}(\rho)}\right) .
$$

Thus in order to conclude the proof of Corollary 1.7], we need to control the length $\ell_{Y}\left(\mathcal{O}_{k Y_{n}(\rho)}\right)$ as a function of $\rho=\epsilon a$. We now reintroduce the rational parameter $a=p / q$, and we will show that for any $0<\frac{p}{q}<1$, we can find $n$ sufficiently large so that

$$
\ell_{Y}\left(\mathcal{O}_{k Y_{n}\left(\frac{p \epsilon}{q}\right)}\right) \leq O\left(\left(\frac{p \epsilon k n}{q}\right)^{\operatorname{codim}(Y, X)}\right),
$$

where the implied constant depends only on $X$ and $\epsilon$. Combining (1.7.5) and (1.7.6) shows (1.7.3), with appropriate divisibility restrictions on $k$ and $n$ according to the rational number $p / q$, and will conclude the proof of Corollary [1.7.

In order to prove (1.7.6), we need to examine how the ideal sheaves $\mathcal{I}_{k Y_{n}(\epsilon)}$ depend upon $k, n$, and $\epsilon$, but only at a general point of $Y$ since we are only interested in controlling the generic length of $\mathcal{O}_{k Y_{n}(\epsilon)}$. Choose $\epsilon^{\prime}$ so that $0<\epsilon^{\prime}-\epsilon \ll \epsilon$. We begin by showing that for fixed $n$, we can find $\beta>0$ such that

$$
\mathcal{I}_{\beta \epsilon^{\prime} k n Y} \subset \mathcal{I}_{k Y_{n}\left(\epsilon^{\prime}\right)} \text { generically for all } k \gg 0 \text {. }
$$

In order to establish (1.7.7), we begin by fixing a $\beta>0$ such that

$$
\mathcal{I}_{\beta \epsilon^{\prime} n Y} \subset \mathcal{I}_{Y_{n}\left(\epsilon^{\prime}\right)}, \text { generically. }
$$

Such a $\beta$ must exist, because by assumption $\mathcal{I}_{Y_{n}\left(\epsilon^{\prime}\right)}$ is generically $\mathcal{I}_{Y}$-primary. We have

$$
\mathcal{I}_{k Y_{n}\left(\epsilon^{\prime}\right)} \supset \mathcal{I}_{Y_{n}\left(\epsilon^{\prime}\right)}^{k} \supset \mathcal{I}_{\beta \epsilon^{\prime} n Y}^{k} \text { generically for all } k>0 .
$$

But, generically, $\mathcal{I}_{\beta \epsilon^{\prime} n Y}^{k}=\mathcal{I}_{\beta \epsilon^{\prime} k n Y}$ for all $k$ sufficiently large, and thus we have established (1.7.7).

Next, in order to allow $\epsilon^{\prime}$ to vary, we will need to let $n$ vary as well. We will show the following: for any positive integer $r$ and for all $k>0$,

$$
\mathcal{I}_{k Y_{n}\left(\epsilon_{1}\right)} \cdot \ldots \cdot \mathcal{I}_{k Y_{n}\left(\epsilon_{r}\right)} \subset \mathcal{I}_{k Y_{r n}\left(\frac{\epsilon_{1}+\ldots+\epsilon_{r}}{r}\right)} \text { generically; }
$$

here the $\epsilon_{i}$ are arbitrary rational numbers less than or equal to $\epsilon^{\prime}$. Choose a birational map $\pi: V \rightarrow X$ so that $V$ is smooth and so that $\pi$ resolves the base loci of the $r+1$ complete linear series

$$
\left|n\left(D-\epsilon_{1} A\right)\right|, \ldots,\left|n\left(D-\epsilon_{r} A\right)\right|,\left|r n\left(D-\frac{\epsilon_{1}+\ldots+\epsilon_{r}}{r} A\right)\right| .
$$

We let $E_{1}+F_{1}, \ldots, E_{r}+F_{r}$, and $E+F$ denote the associated exceptional divisors. The decomposition of the exceptional divisors is determined as follows: we choose 
$E_{1}, \ldots, E_{r}, E$ to contain all exceptional components whose image under $\pi$ is contained in $Y$, and $F_{1}, \ldots, F_{r}, F$ are formed from all remaining components. Thus, no component of $F_{1}, \ldots, F_{r}, F$ is mapped into $Y$ by $\pi$. Then, generically, we have

$$
\begin{array}{r}
\pi_{*} \mathcal{O}_{Y}\left(-E_{i}\right)=\mathcal{I}_{Y_{n}\left(\epsilon_{i}\right)}, \quad 1 \leq i \leq r, \\
\pi_{*} \mathcal{O}_{Y}(-E)=\mathcal{I}_{Y_{r n}\left(\frac{\epsilon_{1}+\ldots+\epsilon_{r}}{r}\right)},
\end{array}
$$

since, as we observed above with the scheme $Z_{n}(\epsilon)$, these ideal sheaves do not depend on the resolution $\pi: V \rightarrow X$.

We claim that

$$
E_{1}+\ldots+E_{r} \geq E
$$

To see this, suppose $s_{i} \in H^{0}\left(X, n\left(D-\epsilon_{i} A\right)\right)$ for $1 \leq i \leq r$. It follows that

$$
s_{1} \otimes \ldots \otimes s_{r} \in H^{0}\left(X, r n\left(D-\frac{\epsilon_{1}+\ldots+\epsilon_{r}}{r} A\right)\right) .
$$

Since the sections $s_{i}$ were arbitrary, lifting (1.7.10) to $V$ establishes that

$$
\left(E_{1}+\ldots+E_{r}\right)+\left(F_{1}+\ldots+F_{r}\right) \geq E+F ;
$$

indeed, (1.7.10) shows that the sum of the common base loci of $\left|n\left(D-\epsilon_{i} A\right)\right|$ is at least as large as the base locus of $\left|r n\left(D-\frac{\epsilon_{1}+\ldots+\epsilon_{r}}{r} A\right)\right|$. By definition of $E_{i}, F_{i}, E$, and $F$ this establishes (1.7.9). We can conclude that for all $k>0$,

$$
\pi_{*}\left(\mathcal{O}_{V}\left(-k E_{1}\right)\right) \cdot \ldots \cdot \pi_{*}\left(\mathcal{O}_{V}\left(-k E_{r}\right)\right) \subset \pi_{*}\left(\mathcal{O}_{V}(-k E)\right),
$$

and this is precisely (1.7.8), with the inclusion holding off of $\operatorname{supp}\left(Y_{n, k}\right) \cap \operatorname{supp}\left(W_{n, k}\right)$.

In order to use (1.7.8) to establish (1.7.7), we apply (1.7.8) as follows. Choose a rational number $\frac{p}{q}<1$ and let

$$
\epsilon_{1}=\ldots=\epsilon_{p}=\epsilon^{\prime}
$$

and

Applying (1.7.8),

$$
\epsilon_{p+1}=\ldots=\epsilon_{q}=\frac{p \epsilon-p \epsilon^{\prime}}{q-p}
$$

$$
\mathcal{I}_{k Y_{n}\left(\epsilon_{1}\right)} \cdot \ldots \cdot \mathcal{I}_{k Y_{n}\left(\epsilon_{q}\right)} \subset \mathcal{I}_{k Y_{q n}\left(\frac{p \epsilon}{q}\right)} \text { generically for all } k>0 .
$$

By the choice of $\epsilon_{1}, \ldots, \epsilon_{q}$ this gives

$$
\mathcal{I}_{k Y_{n}\left(\epsilon^{\prime}\right)}^{p} \subset \mathcal{I}_{k Y_{q n}\left(\frac{p \epsilon}{q}\right)} \text { generically for all } k>0 .
$$

Using (1.7.7), 1.7.11 becomes

$$
\mathcal{I}_{\beta p \epsilon^{\prime} k n Y} \subset \mathcal{I}_{k Y_{q n}\left(\frac{p \epsilon}{q}\right)}, \text { generically for all } k \gg 0 .
$$

Now we will use (1.7.12) in order to estimate lengths and establish (1.7.6), concluding the proof of Corollary 1.7 We have, by (1.7.12) above,

$$
\ell_{Y}\left(\mathcal{O}_{k Y_{q n}\left(\frac{p \epsilon}{q}\right)}\right) \leq \ell_{Y}\left(\mathcal{O}_{\beta p \epsilon^{\prime} k n Y}\right) .
$$

Subsituting $n^{\prime}=q n$ into (1.7.13) gives

$$
\ell_{Y}\left(\mathcal{O}_{k Y_{n^{\prime}}\left(\frac{p \epsilon}{q}\right)}\right) \leq \ell_{Y}\left(\mathcal{O}_{\frac{\beta p \epsilon^{\prime} k n^{\prime} Y}{q}}\right) .
$$


A quick computation shows that

$$
\ell_{Y}\left(\mathcal{O}_{\frac{\beta \epsilon^{\prime} k n Y}{q}}\right)=O\left(\left(\frac{\beta \epsilon^{\prime} p k n^{\prime}}{q}\right)^{\operatorname{codim}(Y, X)}\right) .
$$

This is precisely (1.7.6), with $\epsilon^{\prime}$ in place of $\epsilon$ and with an additional factor of $\beta$ on the right-hand side. Since $\epsilon^{\prime}$ is arbitrarily close to $\epsilon$ and since $\beta$ is a fixed constant depending only on $\epsilon$, this establishes Corollary 1.7.

Proof of Lemma 1.8. Choose $\epsilon_{1}, \epsilon_{2}>\epsilon$ so that $D-\epsilon_{1} A, D+\epsilon_{2} A \in \operatorname{Div}(X) \otimes \mathbf{Q}$. Choose a birational map $\pi: V \rightarrow X$, with $V$ smooth, resolving the base loci of $\left|n\left(D-\epsilon_{1} A\right)\right|$ and $\left|n\left(D+\epsilon_{2} A\right)\right|$ for appropriately divisible $n$. Let $E_{1}$ and $E_{2}$ be the corresponding exceptional divisors. By assumption, $E_{2}$ contains no component dominating $Y$, while $E_{1}$ contains at least one such component. As in the proof of Corollary [1.7, we will write

$$
E_{1}=E_{1}(Y)+F_{1}
$$

where every component of $E_{1}(Y)$ maps into $Y$ and no component of $F_{2}$ is mapped into $Y$ by $\pi$. We claim that

$$
\pi_{*} \mathcal{O}_{V}\left(-k F_{1}\right)=\mathcal{I}_{k W_{n}\left(\epsilon_{1}\right)}, \text { for all } k>0 .
$$

To prove (1.8.1), let $U=X \backslash Y$ and observe that

$$
\pi_{*} \mathcal{O}_{V}\left(-k F_{1}\right)\left|U=\mathcal{I}_{k W_{n}\left(\epsilon_{1}\right)}\right| U \text {. }
$$

But since no component of $F_{1}$ is mapped into $Y$, it follows that $\pi_{*} \mathcal{O}_{V}\left(-k F_{1}\right)$ is the largest ideal sheaf whose restriction to $U$ is equal to $\pi_{*} \mathcal{O}_{V}\left(-k F_{1}\right) \mid U$; since $\mathcal{I}_{k W_{n}\left(\epsilon_{1}\right)}$ was chosen to be maximal, having no embedded components along $\operatorname{supp}(Y)$, this establishes (1.8.1).

Choose a $\pi$-exceptional divisor $F \subset V$, containing no component of $E_{1}(Y)$, such that

$$
F>F_{1} .
$$

Let $\mathcal{J}=\pi_{*} \mathcal{O}_{V}(-F)$. Using (1.8.1) and (1.8.2) establishes that

$$
\mathcal{J}^{k} \subset \mathcal{I}_{k W_{n}\left(\epsilon_{1}\right)}, \quad \forall k>0 .
$$

Moreover, if $r>0$, we have by (1.8.3),

$$
\mathcal{J}^{k r} \subset \mathcal{I}_{k W_{n}\left(\epsilon_{1}\right)}^{r} \subset \mathcal{I}_{k W_{r n}\left(\epsilon_{1}\right)} \text {, for all } r>0,
$$

and this is precisely Lemma 1.8 in the case where $m=0$.

Fix an $m>0$ and choose a positive integer $r_{m}$ and a birational map

$$
\pi_{m}: X_{m} \rightarrow X
$$

with $X_{m}$ smooth, resolving the base loci of the following complete linear series:

$$
\left|\left(2^{m}-1\right) r_{m} n\left(D-\epsilon_{1} A\right)\right|,\left|n\left(D+\epsilon_{2} A\right)\right|,\left|2^{m} r_{m} n\left(D-\epsilon_{1} A+\frac{\epsilon_{1}+\epsilon_{2}}{2^{m}} A\right)\right| .
$$

Let $G_{1}, G_{2}$, and $G^{\prime}$ denote the part of the exceptional divisor in the three cases which does not lie over $Y$. We claim that

$$
G_{1}+r_{m} G_{2} \geq G^{\prime}
$$

As we have seen above after (1.7.10), this will be true provided that for any sections

$$
s_{1} \in H^{0}\left(X,\left(2^{m}-1\right) r_{m} n\left(D-\epsilon_{1} A\right)\right) \quad \text { and } \quad s_{2} \in H^{0}\left(X, n\left(D+\epsilon_{2} A\right)\right)
$$


we have

$$
s_{1} \otimes s_{2}^{\otimes r_{m}} \in H^{0}\left(X, 2^{m} r_{m} n\left(D-\epsilon_{1} A+\frac{\epsilon_{1}+\epsilon_{2}}{2^{m}} A\right)\right),
$$

which is an elementary computation of line bundles. Pushing (1.8.5), multiplied by $k$, down to $X$ and using (1.8.4) yields

$$
\mathcal{J}^{k r_{m}} \cdot \mathcal{I}_{k W_{\left(2^{m}-1\right) r_{m} n\left(-\epsilon_{1}\right)}} \subset \mathcal{I}_{k W_{2^{m} r_{m} n}\left(-\epsilon_{1}+\frac{\epsilon_{1}+\epsilon_{2}}{2^{m}}\right)} \text {, for all } k \gg 0 \text {. }
$$

We now replace $\epsilon_{1}$ with $\beta$, which may necessitate increasing $n$ in order to clear denominators, and observe that

$$
\mathcal{I}_{k W_{l}(a)} \subset \mathcal{I}_{k W_{l}(b)}, \quad \forall k, l,
$$

whenever $a>b$. Thus we conclude from (1.8.6) that for any $\beta<\epsilon$,

$$
\mathcal{J}^{k r_{m}} \cdot \mathcal{I}_{k W_{\left(2^{m}-1\right) r_{m} n(-\beta)}} \subset \mathcal{I}_{k W_{2^{m} r_{m} n}\left(-\beta+\frac{\epsilon}{2^{m}}\right)} \text {, for all } k \gg 0,
$$

provided, as observed above, that $r_{m}$ is sufficiently divisible. This concludes the proof of Lemma 1.8 and hence the proof of Theorem 0.8. If $D \in \operatorname{Div}(X) \otimes \mathbf{Q}$, then the same argument works for $\beta=0$.

\section{A Theorem of Fujita}

In this section we turn to a theorem of Fujita regarding a numerical form of Zariski decomposition. The techniques of this paper are so close to those of [DEL] that it is only natural to apply them in this setting. If $L$ is a big line bundle, we let $v(L)$ denotes its volume as defined in DEL; so $v(L)$ measures the asymptotic growth of $h^{0}(X, n L)$.

Theorem 2.1 (Fujita). Suppose $X$ is a smooth projective variety and $L$ a big line bundle on $X$. For any $\epsilon>0$ there exist a birational map $\pi: Y \rightarrow X$ and $\mathbf{Q}$-divisors $A$ and $E$ such that

$$
\pi^{*} L \equiv A+E,
$$

where $A$ is ample and $v(A) \geq v(L)-\epsilon$.

We will attack this problem using the language of moving Seshadri constants developed in $§ 1$. Consider the following inductive construction. Choose a point

$$
x_{1} \in X \backslash \operatorname{BS}(L)
$$

such that $\epsilon_{m}\left(x_{1}, L\right)$ is maximal. Next choose $\delta_{1} \ll \epsilon_{m}\left(x_{1}, L\right)$ so that

$$
\epsilon_{1}=\epsilon_{m}\left(x_{1}, L\right)-\delta_{1} \in \mathbf{Q} \text {. }
$$

Let $\pi_{1}: X_{1} \rightarrow X$ be the blow-up of $x_{1}$ with exceptional divisor $E_{1}$, and let

$$
L_{1}=\pi_{1}^{*} L\left(-\epsilon_{1} E_{1}\right) .
$$

Next we choose a point $x_{2}$ where $\epsilon_{m}\left(x_{2}, L_{1}\right)$ is maximal, and let $\delta_{2} \ll \epsilon_{m}\left(x_{2}, L_{1}\right)$, so that $\epsilon_{2}=\epsilon_{m}\left(x_{2}, L_{1}\right)-\delta_{2} \in \mathbf{Q}$. Let $\pi_{2}: X_{2} \rightarrow X$ be the blow-up of $X$ at both $x_{1}$ and $x_{2}$, and let

$$
L_{2}=\pi_{2}^{*} L\left(-\epsilon_{1} E_{1}-\epsilon_{2} E_{2}\right) .
$$

This procedure can be repeated inductively to yield pairs $\left(X_{n}, L_{n}\right)$ for all positive $n$. 
Lemma 2.2. Suppose $\eta$ is a general point of $X$, which we also view as a point of $X_{n}$. Then

$$
\lim _{n \rightarrow \infty} \epsilon_{m}\left(\eta, L_{n}\right)=0
$$

Proof of Lemma 2.2. If $\lim _{n \rightarrow \infty} \epsilon_{m}\left(\eta, L_{n}\right) \neq 0$, this means that there exists $\alpha>0$ such that, for $k$ suitably large and divisible, $k L$ generates $k \alpha$-jets at arbitrarily many points $x_{1}, x_{2}, \ldots$, assuming that $\delta_{i}$ is sufficiently small relative to $\epsilon_{i}$. This is clearly impossible, since $v(L)$ bounds the number of such points.

Lemma 2.3. Let $D$ be a very ample line bundle on $X$ and assume that $L_{n}\left(-\alpha \pi_{n}^{*} D\right)$ is not in the effective cone. Then

$$
h^{0}\left(X_{n}, k L_{n}\right) \leq \alpha O\left(k^{\operatorname{dim} X}\right),
$$

with the implied constant depending only on $D$ and $L$.

Proof of Lemma 2.3. Consider the exact sequence

$$
0 \rightarrow H^{0}\left(X_{n}, k L_{n}\left(-\alpha \pi_{n}^{*} D\right)\right) \rightarrow H^{0}\left(X_{n}, k L_{n}\right) \rightarrow H^{0}\left(k \alpha \pi_{n}^{*} D, k L_{n}\right),
$$

where $k$ is sufficiently large and divisible and where $k \alpha \pi_{n}^{*} D$ is represented by the pull-back of $k \alpha$ general smooth hypersurfaces linearly equivalent to $D$. Then we obtain

$$
\begin{aligned}
h^{0}\left(X_{n}, k L_{n}\right) & \leq k \alpha h^{0}\left(\pi_{n}^{*} D, k L_{n}\right) \\
& =k \alpha h^{0}(D, k L) \\
& =\alpha O\left(k^{\operatorname{dim} X}\right) .
\end{aligned}
$$

In the last formula, the implied constant clearly depends only on $D$ and $L$.

Proof of Theorem [2.1. Choose $\alpha>0$. By Lemma 2.2 we can find $n$ so that $L_{n}-$ $\alpha \pi_{n}^{*} D$ is not in the effective cone. Fix $k_{0}$ so that $\left|k_{0} L\right|$ generates $k_{0} \epsilon_{i}$ jets at $x_{i}$ for $1 \leq i \leq n$; we assume here that $k_{0}$ is sufficiently divisible so that $k_{0} \epsilon_{i} \in \mathbf{Z}$ for all $i$. Thus the sequence

$$
\begin{aligned}
0 & \rightarrow H^{0}\left(X, m k_{0} L \otimes \bigcap_{i=1}^{n} m_{x_{i}}^{m k_{0} \epsilon_{i}}\right) \rightarrow H^{0}\left(X, m k_{0} L\right) \\
& \rightarrow H^{0}\left(m k_{0} L \otimes \mathcal{O}_{X} / \bigcap_{i=1}^{n} m_{x_{i}}^{m k_{0} \epsilon_{i}}\right) \rightarrow 0
\end{aligned}
$$

is exact for all positive integers $m$; here $m_{x_{i}}$ is the maximal ideal sheaf of the point $x_{i}$. Also, by construction and Lemma 2.3

$$
\begin{aligned}
h^{0}\left(X, m k_{0} L \otimes \bigcap_{i=1}^{n} m_{x_{i}}^{m k_{0} \epsilon_{i}}\right) & =h^{0}\left(X_{n}, m k_{0} L_{n}\right) \\
& \leq \alpha c\left(m k_{0}\right)^{\operatorname{dim} X}
\end{aligned}
$$

with $c$ depending only on $D$ and $L$. Let $\pi: Y \rightarrow X$ be a birational map such that $Y$ is smooth and

$$
\pi^{*} k_{0} L \sim M+F
$$

with $M$ base point free. Then $v\left(M / k_{0}\right) \geq v(L)-\alpha c$. Since $\alpha$ was arbitrary, this establishes Theorem [2.1; note that here $M / k_{0}$ is only big and nef, rather than ample, but this can be changed with an arbitrarily small perturbation. 
Finally, we would like to pose a difficult and important question, which we will phrase as a conjecture. According to Theorem 0.8 , if $D$ is effective and $A$ ample, then $D-\alpha A$ reaches the boundary of the effective cone exactly when

$$
\epsilon_{m}(\eta, D-\alpha A)=0
$$

for a general point $\eta$. Our question then is the following:

Conjecture 2.4. Suppose $D \in \operatorname{Div}(X) \otimes \mathbf{Q}$ is a divisor class on the boundary of the effective cone. Then $\epsilon(\eta, D)=0$ for a general point $\eta \in X$.

Using the results of this paper, one can establish under the hypotheses of Conjecture 2.4 that

$$
\epsilon_{m}(\eta, D)=0
$$

but it is not clear whether or not $\epsilon_{m}(\eta, D)=\epsilon(\eta, D)$. If the answer is yes, then one has a numerical criterion to determine the effective cone.

Conjecture 2.4 essentially boils down to the following concrete, computational question. Suppose $L$ is an effective line bundle, and suppose $\pi_{n}: Y_{n} \rightarrow X$ resolves the base locus of $|n L|$ with $Y_{n}$ smooth. Writing $\left(\pi_{n}\right)^{*} n L=M_{n}+E_{n}$, is it true that

$$
\lim _{n \rightarrow \infty} \frac{M_{n}^{\operatorname{dim} X-1} \cdot E_{n}}{n^{\operatorname{dim} X}}=0 ?
$$

In other words, does the pair $\left(M_{n}, E_{n}\right)$ approximate a Zariski decomposition not only in the sense of Theorem 2.1 but also in a stronger sense of becoming closer and closer to being perpendicular?

\section{REFERENCES}

[DEL] J.-P. Demailly, L. Ein, and R. Lazarsfeld, A subadditivity property of multiplier ideals, Michigan Math. J. 48 (2000), 137-156. MR 2002a:14016

[ELN] L. Ein, R. Lazarsfeld, and M. Nakamaye, Zero Estimates, Intersection Theory, and a Theorem of Demailly, in Andreatta and Peternell, eds., Higher Dimensional Complex Varieties, de Gruyter, 1996, pp. 183-208. MR 99c:14006

[EV] H. Esnault and E. Viehweg, Dyson's Lemma for polynomials in several variables (and the Theorem of Roth), Invent. Math., 78, 1984, pp. 445-490. MR 86e:11053

[Fu] W. Fulton, Intersection Theory, Springer-Verlag, 1984. MR 85k:14004

[KMM] Y. Kawamata, K. Matsuda, and K. Matsuki, Introduction to the Minimal Model program, In: Oda, T. (ed.) Algebraic Geometry. Proc. Symp., Sendai, 1985, (Adv. Stud. Pure Math.,10, pp. 283-360). MR 89e:14015

[N] M. Nakamaye, Stable Base Loci of Linear Series, Mathematische Annalen, 318, 2000, pp. 837-847. MR 2002a:14008

Department of Mathematics and Statistics, University of New Mexico, Albuquerque, New Mexico 87131

E-mail address: nakamaye@math.unm.edu 